\title{
An essential role of RNF187 in Notch1 mediated metastasis of hepatocellular carcinoma
}

\author{
Lei Zhang ${ }^{1}$, Jiewei Chen², Juanjuan Yong ${ }^{3}$, Liang Qiao ${ }^{4}$, Leibo Xu ${ }^{1 *}$ and Chao Liu ${ }^{1 *}$
}

\begin{abstract}
Background: Aberrant activation of Notch signaling has been causally linked to the metastasis of hepatocellular carcinoma (HCC), however the underlying molecular mechanisms are still poorly understood. RING finger protein 187 (RNF187) was recently revealed to be a driver of several cancers, but its expression pattern and biological function in HCC are unknown.
\end{abstract}

Methods: The expression levels of Notch1 and RNF187 were assessed in two independent cohorts of HCC tissues, and modulation of Notch1 in HCC cells was performed to explore the regulatory role of Notch1 in HCC metastasis. RNA-sequencing (RNA-seq), bioinformatics analysis, luciferase reporter analysis, and chromatin immunoprecipitation assay (ChIP) were used to clarify the relationship between Notch1 signaling and its potential target Ring finger protein 187 (RNF187). Gain- and loss-of-function studies were used to dissect the role of Notch1-RNF187 signaling in promoting HCC metastasis. The impact of Notch1-RNF187 activity in determining clinical prognosis for HCC patients was evaluated by multivariate Cox regression.

Results: By RNA-seq, luciferase reporter analysis, and ChIP assay, RNF187 was confirmed to be a direct transcriptional target of Notch1, as Notch1 could activate RNF187 promoter whereas the pro-migratory and pro-invasive effects of Notch1 were significantly attenuated by RNF187 knockdown. Meanwhile, RNF187 silencing could attenuate the Notch1-dependent epithelial-mesenchymal transition (EMT). Moreover, overexpression of RNF187 counteracted the inhibitory effect of Notch1 knockdown on cancer progression. Importantly, HCC patients with high level of hepatic Notch1 expression had shorter disease-free survival (DFS) than those with low level of hepatic Notch1 expression. Furthermore, patients with high level of Notch1 and RNF187 co-expression showed the shortest DFS. The expression level of Notch1 and RNF187 was an independent prognostic factor for HCC.

Conclusions: For the first time we identified that RNF187 is an essential factor for Notch1 to promote invasion and metastasis of HCC. Of highly clinical relevance, we found that activation of Notch1-RNF187 correlates with a worse prognosis of HCC patients. These findings provide a solid foundation for developing novel strategies to tackle HCC metastasis.

Keywords: Hepatocellular carcinoma, RNF187, Notch signaling, Metastasis

\footnotetext{
*Correspondence: xuleibo3@mail.sysu.edu.cn; Liuchao3@mail.sysu.edu.cn

1 Guangdong Provincial Key Laboratory of Malignant Tumor Epigenetics and

Gene Regulation and Department of Biliary-Pancreatic Surgery, Sun Yat-sen

Memorial Hospital, Sun Yat-sen University, 107 Yan Jiang West Rd,

Guangzhou 510120, China

Full list of author information is available at the end of the article
}

(c) The Author(s). 2019 Open Access This article is distributed under the terms of the Creative Commons Attribution 4.0 International License (http://creativecommons.org/licenses/by/4.0/), which permits unrestricted use, distribution, and reproduction in any medium, provided you give appropriate credit to the original author(s) and the source, provide a link to the Creative Commons license, and indicate if changes were made. The Creative Commons Public Domain Dedication waiver (http://creativecommons.org/publicdomain/zero/1.0/) applies to the data made available in this article, unless otherwise stated. 


\section{Background}

Hepatocellular carcinoma (HCC) is one of the most prevalent and life-threatening malignancies globally [1]. Metastasis significantly contributes to the high mortality of HCC patients after surgical curative therapy [2, 3]. HCC metastasis occurs both inside and outside the liver. Extrahepatic metastasis have been reported in 13.5-42\% of HCC patients [4]. The median survival time of $\mathrm{HCC}$ patients with extrahepatic metastasis are only 4.9-7 months [4-6]. Thus, it is of great significance to further identify the potential mechanisms of HCC metastasis, which may contribute to the detection and intervention of HCC metastasis in early stage and formulate novel approaches to treat metastatic HCC. Notch family is evolutionarily conserved and plays fundamental roles in diverse biological processes, ranging from selfreorganization to the differentiation [7]. Altered Notch pathway not only contributes to multisystemic developmental defects, but also to the development and progression of many types of cancer [8-10]. Although abnormal expression of the Notch pathway has been demonstrated in $\mathrm{HCC}[11,12]$, the molecular pathways governing metastatic $\mathrm{HCC}$ are not fully characterized and requires further investigation.

Ubiquitination is one of the most abundant posttranslational modifications and is involved in basic cellular processes [13]. Its dysfunction is associated with a series of diseases, particularly in tumorigenesis [14]. E3 ubiquitin ligases confer the substrate specificity and selectivity of the ubiquitin-conjugating system, and are of critical importance in this process [15]. RING finger protein 187 (RNF187), also known as RING domain AP-1 co-activator1 (RACO-1), is a RING domain-containing ubiquitin E3 ligase $[16,17]$. RNF187, a c-Jun co-activator to growth factor signal, is essential for AP-1 function in cell proliferation [17]. A recent study reported that RNF187 promotes HCC metastasis through inducing epithelial-mesenchymal transition (EMT) of HCC cells [18]. Increased expression of RNF187 has been correlated with poor survival in many cancers $[18,19]$. However, the detailed molecular mechanism of how RNF187 promotes HCC metastasis has not been elucidated.

In the present study, using RNA-sequencing (RNAseq), we found that Notch1 up-regulated RNF187 expression in HCC cells, and Notch1-mediated HCC metastasis was attenuated when RNF187 was knocked down, whereas overexpression of RNF187 counteracted the inhibition of cancer progression mediated by Notch1 knockdown, suggesting that RNF187 is essential for Notch1-mediated HCC metastasis. Moreover, we found that Notch1-RNF187 association correlates with the prognosis of HCC patients, which may provide a promising strategy for the treatment of Notch1driven HCC metastasis.

\section{Materials and methods}

\section{Tissue samples}

Two cohorts of HCC patients were enrolled in our study. A total of 150 pairs of paraffin-embedded HCC specimens and corresponding adjacent non-tumor liver tissues (ANLTs) were collected as cohort I from 150 patients who have received curative surgical therapy at the Sun Yat-sen Memorial Hospital (Guangzhou, China) from 2013 to 2015. Another group of tissues samples from 67 patients with HCC treated at the Sun Yat-sen University Cancer Center were included in the cohort II and were used for microarrays chips. Ethics approval of this study was granted by the Ethics Committee of the Sun Yat-sen University. Disease-free survival (DFS) were calculated from the date of liver resection to the date when recurrence or metastasis was detected or the last follow-up visit.

\section{Animal studies}

All animal experiments were approved by the Institutional Animal Care and Use Committee of Sun Yat-sen University (Guangzhou, China). Four-weeks-old male $\mathrm{BALB} / \mathrm{C}$ nude mice (Beijing Vital River Laboratory Animal Technology Co., Ltd., China) were housed under a dedicated SPF facility on a $12 \mathrm{~h}$ light/dark cycle, and were cared for according to the Guide of the Care and Use of Laboratory Animals (National Institutes of Health publication nos. 80-23, revised 1996) and the institutional ethical guidelines for animal experiments.

For spontaneous metastasis assays, HCCLM6-luciferase cells $\left(5 \times 10^{6}\right)$ with or without Notch1 knockdown were injected into the left hepatic lobe of nude mice. The in vivo tumor metastasis was detected by bioluminescence with the IVIS imagining system (Caliper Life Sciences, Hopkinton, MA, USA) after masking the signal from primary xenografts. In the lung colonization assays, mice were injected intravenously with HCCLM6-shNotch1 cells, PLC/PRF5-Notch1 and their control cells $\left(2 \times 10^{6}\right)$. Mice were euthanized at the indicated time after xenograft implantation, and livers and/or lungs were fixed in formalin and embedded in paraffin using a routine method. Lung metastasis were validated on $\mathrm{H} \& \mathrm{E}$ stained sections and lung metastatic foci were counted.

\section{Statistical analysis}

Statistical analyses were performed using SPSS (version 16.0) and Graphpad Prism (version 7.0). Quantitative data were compared using the Student's t-test or oneway analysis of variance (ANOVA) for at least three groups [20]. Categorical data were analyzed using the Chi-square test. Disease-free survival (DFS) curves were determined by the Kaplan-Meier and log-rank test [21]. The cox proportional hazards regression model was used 
to verify the independent risk factors based on the variables selected in univariate and multivariate analysis. Gene ontology (GO) analysis was performed when gene sets were submitted to the DAVID website (http://david. abcc.ncifcrf.gov/home.jsp). A two-tailed $P$ value of less than 0.05 was considered as statistically significant. More details of the materials and methods in this study are described in the Additional file 1.

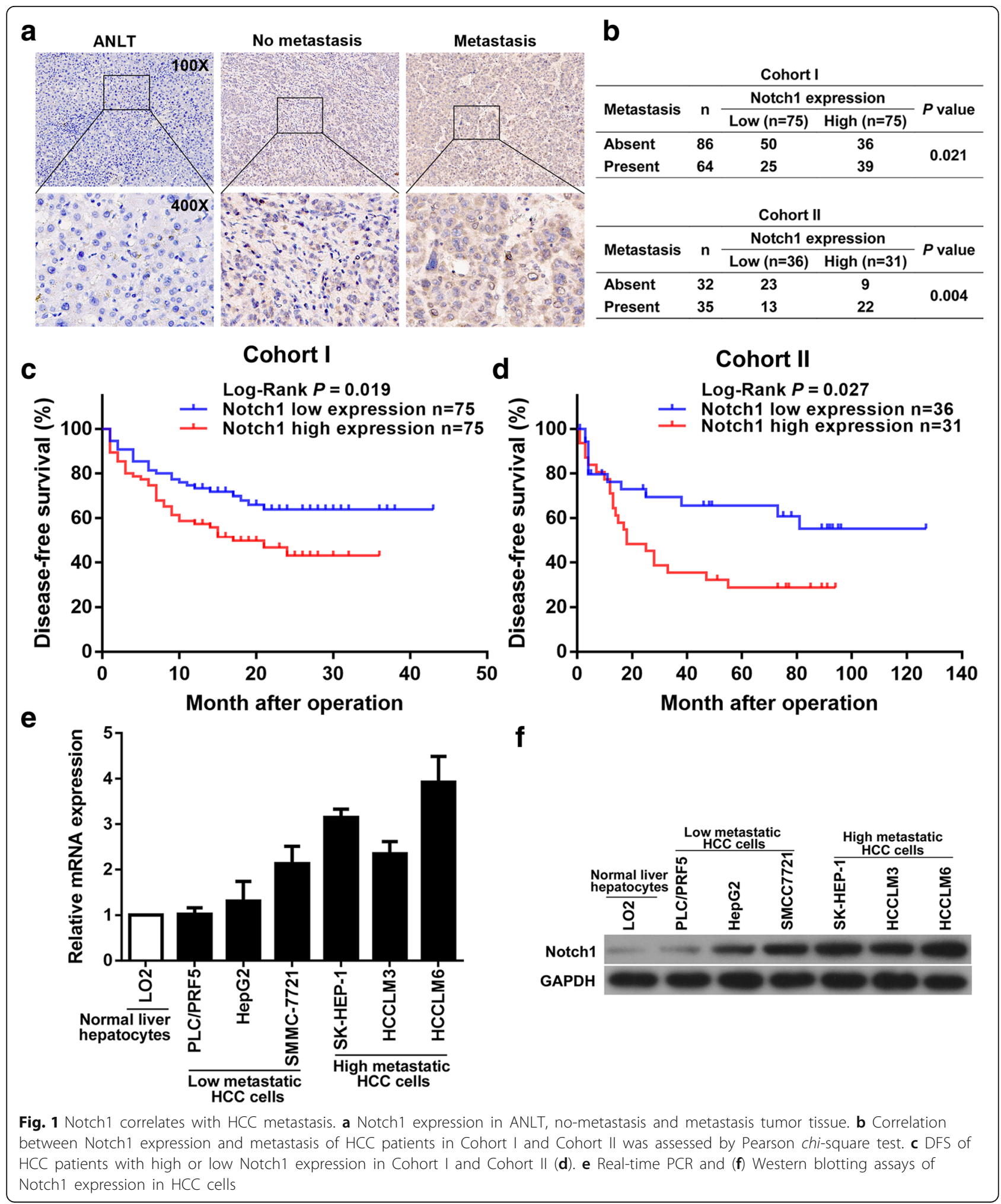




\section{Results}

\section{Notch1 significantly correlates with HCC metastasis}

Immunohistochemistry (IHC) staining indicated that metastasis $\mathrm{HCC}$ tissues had the highest intensities of Notch1 staining, comparing with non-metastasis HCC tissues and ANLTs, (Fig. 1a). The correlation of Notch1 expression with $\mathrm{HCC}$ metastasis were estimated. It showed that high Notch1 expression was significantly correlated with metastasis in HCC patients from both cohort I and cohort II (Fig. 1b). Then, we analyzed the correlation of Notch1 expression with clinicopathological features of HCC patients. In the cohort I, it showed that high Notch1 expression was closely correlated with microvascular invasion in HCC patients, as shown in Table 1. Next, further survival analysis in cohort I showed that high Notch1 expression group had shorter DFS time than low Notch1 expression group (Fig.1c). Survival analysis for the cohort II also demonstrated this point (Fig. 1d).

Next, Real-time polymerase chain reaction (Real-time PCR) and Western blotting assays were performed to analyze the expression of Notch1 in HCC cells. Compared with the human normal liver LO2 cell, mRNA level of Notch1 was highly expressed in HCC cells (Fig. 1e). Increased protein expression level of Notch1 was further confirmed by Western blotting assays (Fig. 1f). Notably, the expression level of Notch1 in high-metastasis potential cell lines, such as SK-HEP-1, HCCLM3 and HCCLM6 was higher than that in low-metastasis potential cell lines
PLC/PRF5, HepG2 and SMMC7721. Taken together, using multiple and complementary approaches in multiple samples including patient HCC tissues and HCC cell lines of low (PLC/PRF5, HepG2 and SMMC7721) and high (SK-HEP-1, HCCLM3 and HCCLM6) metastatic potentials, as well as using the survival analysis, we clearly demonstrated that increased expression of Notch1 correlates with increased metastasis of HCC.

\section{Notch1 promotes HCC cell invasion in vitro and metastasis in vivo}

According to Notch1 expression level, we up-regulated Notch1 in low Notch1 expressing PLC/PRF5 cells by pCMV vector, named PLC/PRF5-pCMV-Notch1. High Notch1 expressing HCCLM6 cells were chosen to knock down Notch1 using small interfering RNA (siRNA), labeled HCCLM6-siRNA. The knockdown efficiency of three siRNAs for Notch1 was confirmed at both mRNA and protein levels through comparison with negative control (sicontrol) (Additional file 2: Figure S1). Among the three siRNAs tested, siRNA2 generated the most consistent knockdown results and was thus selected for further experiments. Next, we performed a variety of in vitro assays to evaluate the effect of Notch1 overexpression on HCC cell proliferation, migration and invasion. The results showed that Notch1 overexpression significantly promoted PLC/PRF5 proliferation by CCK8 assays (Fig. 2a). Colony formation assays confirmed the effect of Notch1 overexpression on cell proliferation

Table 1 Association of Notch1 expression with clinicopathological factors of the HCC patients in cohort I and cohort II

\begin{tabular}{|c|c|c|c|c|c|c|c|c|c|}
\hline \multicolumn{2}{|c|}{ Clinicopathological variables } & \multicolumn{4}{|c|}{ Cohort I } & \multicolumn{4}{|c|}{ Cohort II } \\
\hline & & \multirow{3}{*}{$\begin{array}{l}n \\
122\end{array}$} & \multicolumn{2}{|c|}{ Notch1 expression } & \multirow{3}{*}{$\begin{array}{c}{ }^{\mathrm{a}} \mathrm{P} \text { value } \\
1.000\end{array}$} & \multirow{3}{*}{$\begin{array}{l}n \\
54\end{array}$} & \multicolumn{2}{|c|}{ Notch1 expression } & \multirow[t]{2}{*}{${ }^{a} p$ value } \\
\hline & & & $\operatorname{Low}(n=75)$ & High $(n=75)$ & & & $\operatorname{Low}(n=36)$ & $\operatorname{High}(n=31)$ & \\
\hline \multirow[t]{2}{*}{ Sex } & Male & & 61 & 61 & & & 31 & 23 & 0.219 \\
\hline & Female & 28 & 14 & 14 & & 13 & 5 & 8 & \\
\hline \multirow[t]{2}{*}{ Age, years } & $\leq 50$ & 63 & 35 & 28 & 0.247 & 38 & 17 & 21 & 0.091 \\
\hline & $>50$ & 87 & 40 & 47 & & 29 & 19 & 10 & \\
\hline \multirow[t]{2}{*}{ Serum AFP, ng/mL } & $\leq 20$ & 63 & 33 & 30 & 0.620 & 23 & 12 & 11 & 0.853 \\
\hline & $>20$ & 87 & 42 & 45 & & 44 & 24 & 20 & \\
\hline \multirow[t]{2}{*}{ Tumor nodule number } & Solitary & 123 & 60 & 63 & 0.524 & 52 & 28 & 24 & 0.972 \\
\hline & Multiple & 27 & 15 & 12 & & 15 & 8 & 7 & \\
\hline \multirow[t]{2}{*}{ Maximal tumor size, $\mathrm{cm}$} & $\leq 5$ & 69 & 35 & 34 & 0.870 & 37 & 21 & 16 & 0.581 \\
\hline & $>5$ & 81 & 40 & 41 & & 30 & 15 & 15 & \\
\hline \multirow[t]{2}{*}{ Microvascular invasion } & Absent & 68 & 40 & 28 & 0.049 & 39 & 24 & 15 & 0.130 \\
\hline & Present & 82 & 35 & 47 & & 28 & 12 & 16 & \\
\hline \multirow[t]{2}{*}{ Tumor differentiation } & Well & 19 & 9 & 10 & 0.806 & 22 & 14 & 8 & 0.256 \\
\hline & Moderately or poorly & 131 & 66 & 65 & & 45 & 22 & 23 & \\
\hline \multirow[t]{2}{*}{ TNM stage } & | and || & 97 & 49 & 48 & 0.864 & 47 & 26 & 21 & 0.689 \\
\hline & III and IV & 53 & 26 & 27 & & 20 & 10 & 10 & \\
\hline
\end{tabular}

${ }^{a}$ Pearson chi-square test was used for comparison between subgroups. Significant results $(P<0.05)$ are given in bold 


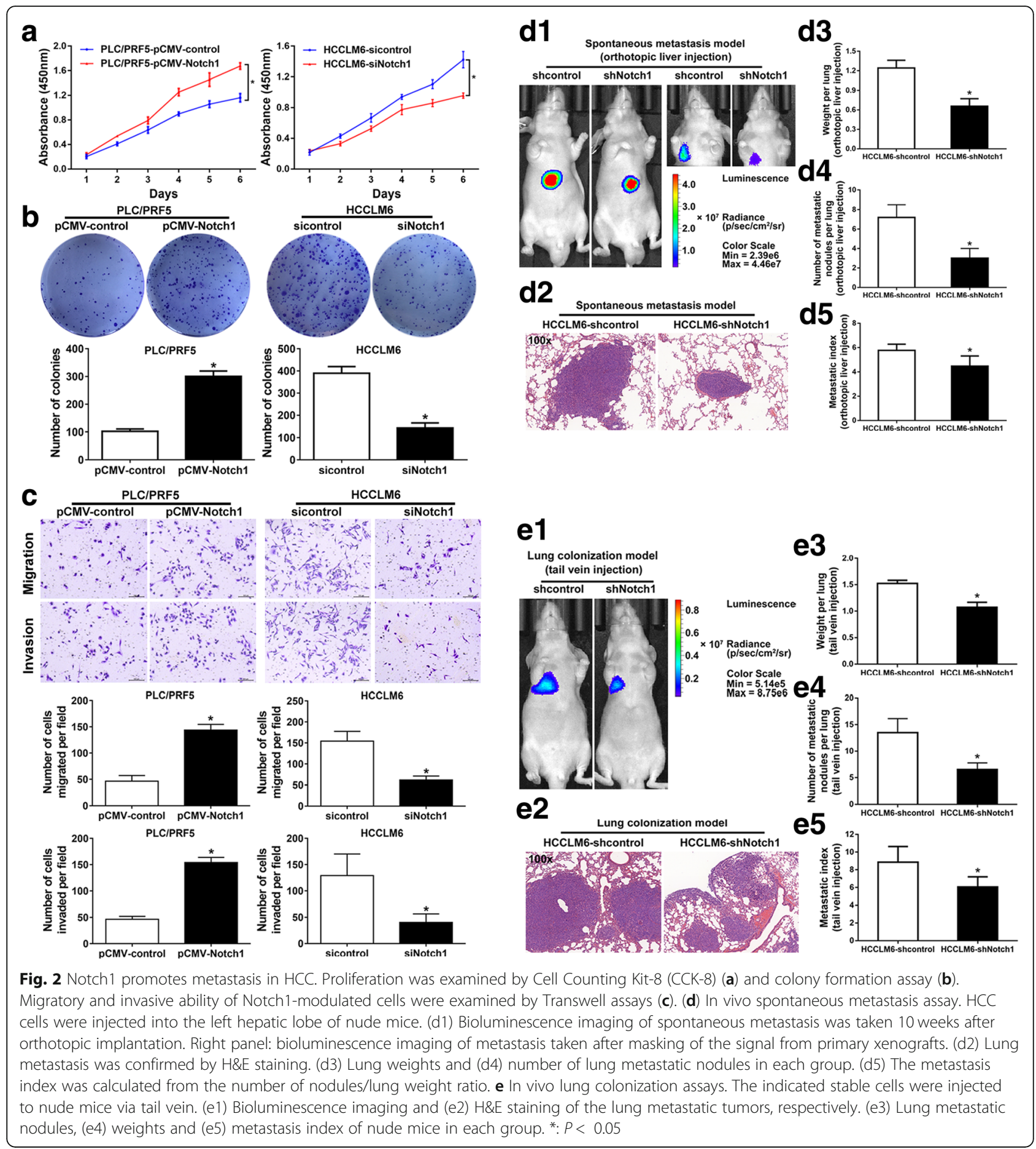

(Fig. 2b). Transwell assays showed that cell migration and invasion were significantly enhanced by Notch1 upregulation in PLC/PRF5 cells (Fig. 2c). Then we assessed the effect of Notch1 downregulation on HCCLM6 cells. The CCK- 8 and colony formation assays showed that Notch1 knockdown significantly suppressed HCCLM6 cell proliferation (Fig. 2a and b). Results from transwell assays demonstrated that Notch1 downregulation impaired cell migration and invasion of HCCLM6 cells (Fig. 2c). These results indicated that Notch1 could be an influential role in the regulation of functions of HCC cell.

To study the role of Notch1 in metastasis of HCC, we manipulated Notch1 stable expression in HCC cells. Ectopic Notch1 was expressed in PLC/PRF5 cells, named as PLC/PRF5-Notch1. Meanwhile, short hairpin RNA 


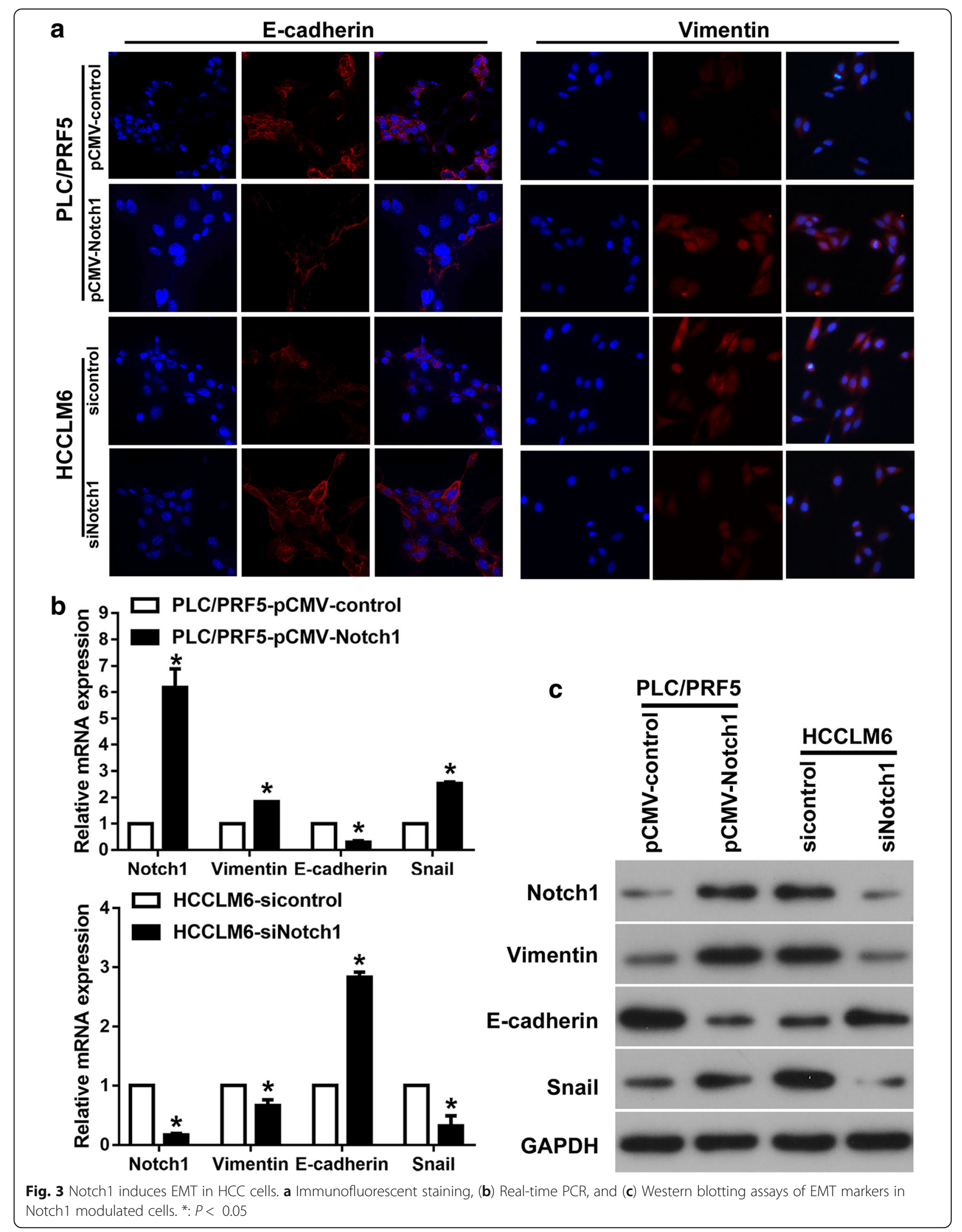




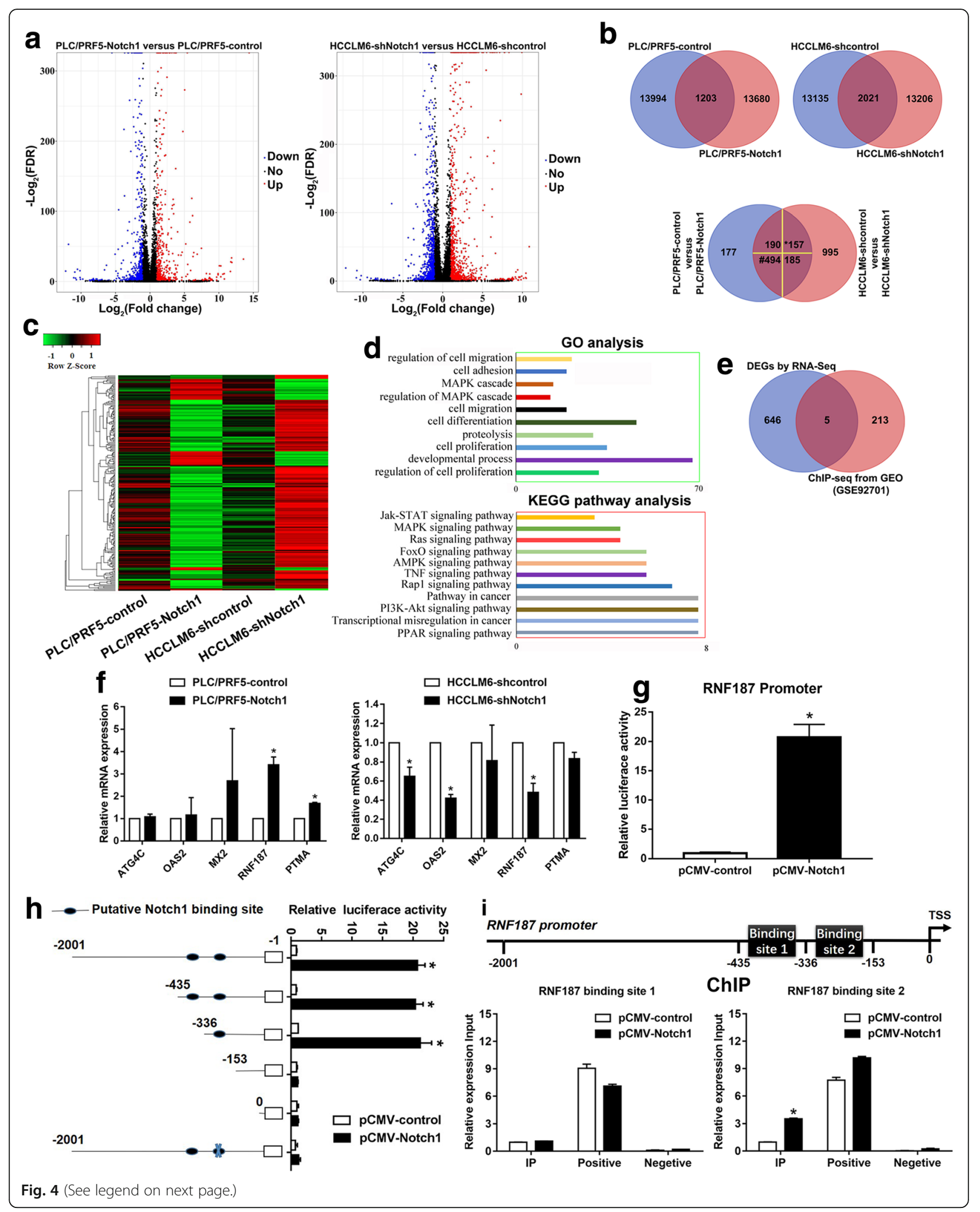




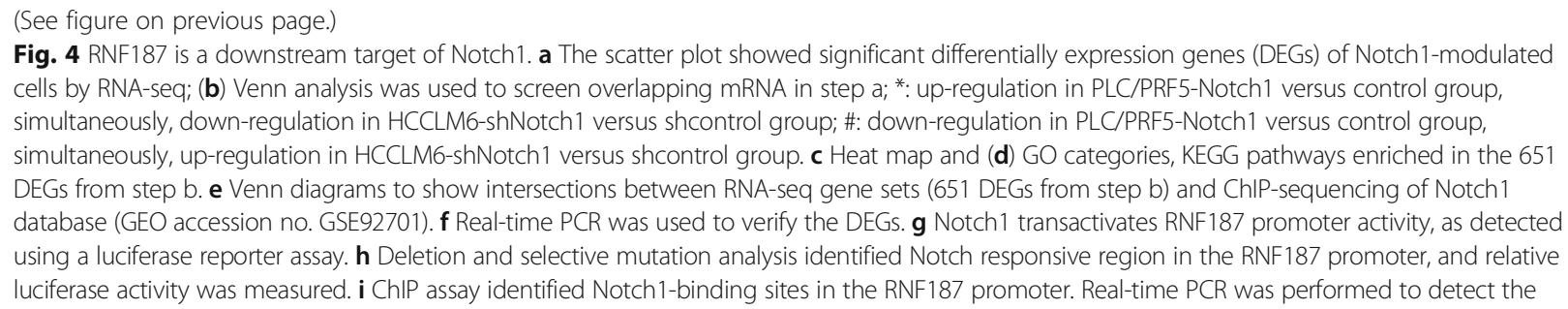

(shRNA), sharing the same sequence with Notch1-siRNA2, was applied to knockdown Notch1 expression in HCCLM6 cells, named as HCCLM6-shNotch1. Expression level of Notch1 was confirmed by Real-time PCR and Western blotting assays (Additional file 3: Figure S2). Then, we established orthotopic xenograft tumor model and vein tail model, as previously described [22]. The HCCLM6shNotch1 cells with stable knockdown of Notch1, as well as control cells, were implanted into nude mice to observe the metastasis of tumor cells in vivo. Orthotopic xenograft tumor model revealed that Notch1 knockdown inhibited tumor metastasis in vivo (Fig. 2d). Bioluminescence imaging showed that knockdown of Notch1 expression strongly inhibited the metastasis of HCCLM6 cells (Fig. 2 d1), which was further confirmed by H\&E-stained sections (Fig. $2 \mathrm{~d} 2$ ). We further detected the metastatic nodules in lungs and lung weight (Fig. $2 \mathrm{~d} 3$ and d4). The lung metastasis rates in nude mice with tumors derived from HCCLM6shNotch1 cells were significantly lower than that derived from control group (Fig. 2 d5). We also performed lung metastasis model by injecting cells into the lateral tail vein. As assessed by bioluminescence imaging (Fig. 2 e1) and H\&E staining (Fig. 2 e2), Notch1 downregulation resulted in a decrease of numbers of lung metastatic nodules (Fig. 2 e3) and lung weight (Fig. 2 e4), as well as metastasis rates (Fig. 2 e5). The PLC/PRF5-Notch1 cells, as well as control cells, were implanted into nude mice to observe the metastasis of tumor cells in vivo through vein tail model. As assessed by H\&E staining (Additional file 4: Figure S3a), Notch1 upregulation resulted in an increase of lung weight (Additional file 4: Figure S3b), numbers lung metastatic nodules (Additional file 4: Figure S3c), and metastasis rates (Additional file 4: Figure S3d). From the above findings, our studies showed that Notch1 could promote metastasis in HCC.

\section{Notch1 induces EMT in HCC cells}

To investigate whether Notch1 promotes HCC invasion and migration by EMT, we evaluated epithelial and mesenchymal markers in HCC. Ectopic expression of Notch1 in PLC/PRF5 cells resulted in the decreased expression of E-cadherin and increased expression of Vimentin as evidenced by Immunofluorescence (IF); whereas Notch1 downregulation increased E-cadherin expression and decreased Vimentin expression in HCCLM6 cells (Fig. 3a). Consistent with the above findings, Notch1 upregulated cells showed decreased E-cadherin and increased Vimentin and Snail (EMT transcriptional factor) expressions at both mRNA and protein levels, whereas Notch1 downregulation induced the inverse results (Fig. 3b and c).

\section{RNF187 is a direct transcriptional target of Notch1}

To further delineate the molecular basis by which Notch1 promotes metastasis in HCC, whole genome transcriptome analysis on PLC/PRF5-Notch1, HCCLM6shNotch1, and their control cells were conducted using RNA-seq (Fig. 4a). Statistical analysis allowed the screening out of Differentially Expression Genes (DEGs) that exhibited highly significant differences according to the following criteria: DEGs increased in PLC/PRF5-Notch1 versus control group and simultaneously decreased in HCCLM6-shNotch1 versus shcontrol group; and vice versa, the expression of DEGs reduced in PLC/PRF5Notch1 versus control group and simultaneously increased in HCCLM6-shNotch1 and shcontrol group. By defining a threshold of the cut-off as more than 1.5-fold change, 651 DEGs were screened (Fig. 4b. Additional file 5: Table S4), which mainly enriched regulation of cell migration, adhesion, etc. by Gene Ontology (GO) analysis, as shown in Fig. 4c-d. Kyoto encyclopedia of genes and genomes (KEGG) analysis showed that they activate pathways involved in PI3K-AKt pathway, transcriptional misregulation in cancer, etc. (Fig. 4d).

Next, we downloaded ChIP-sequencing database of Notch1 binding from GEO database (accession no. GSE92701) to further explore the underlying target genes of Notch1. Further Venn analyses with the above RNA-seq results showed that OAS2, ATG4C, RNF187, PTMA, and MX2 may potentially be direct transcriptional targets of Notch1 (Fig. 4e). Changes in these potential target genes were further confirmed by Real-time PCR (Fig. 4f). The results showed that Notch1 upregulation increased the expression of RNF187 and PTMA, and whereas Notch1 downregulation decreased ATG4C, OAS2, and RNF187 expression. Of particular significance was RNF187, which was upregulated 3.4-fold in response to Notch1 overexpression. Then we performed bioinformatic prediction of Notch1 transcription factor 


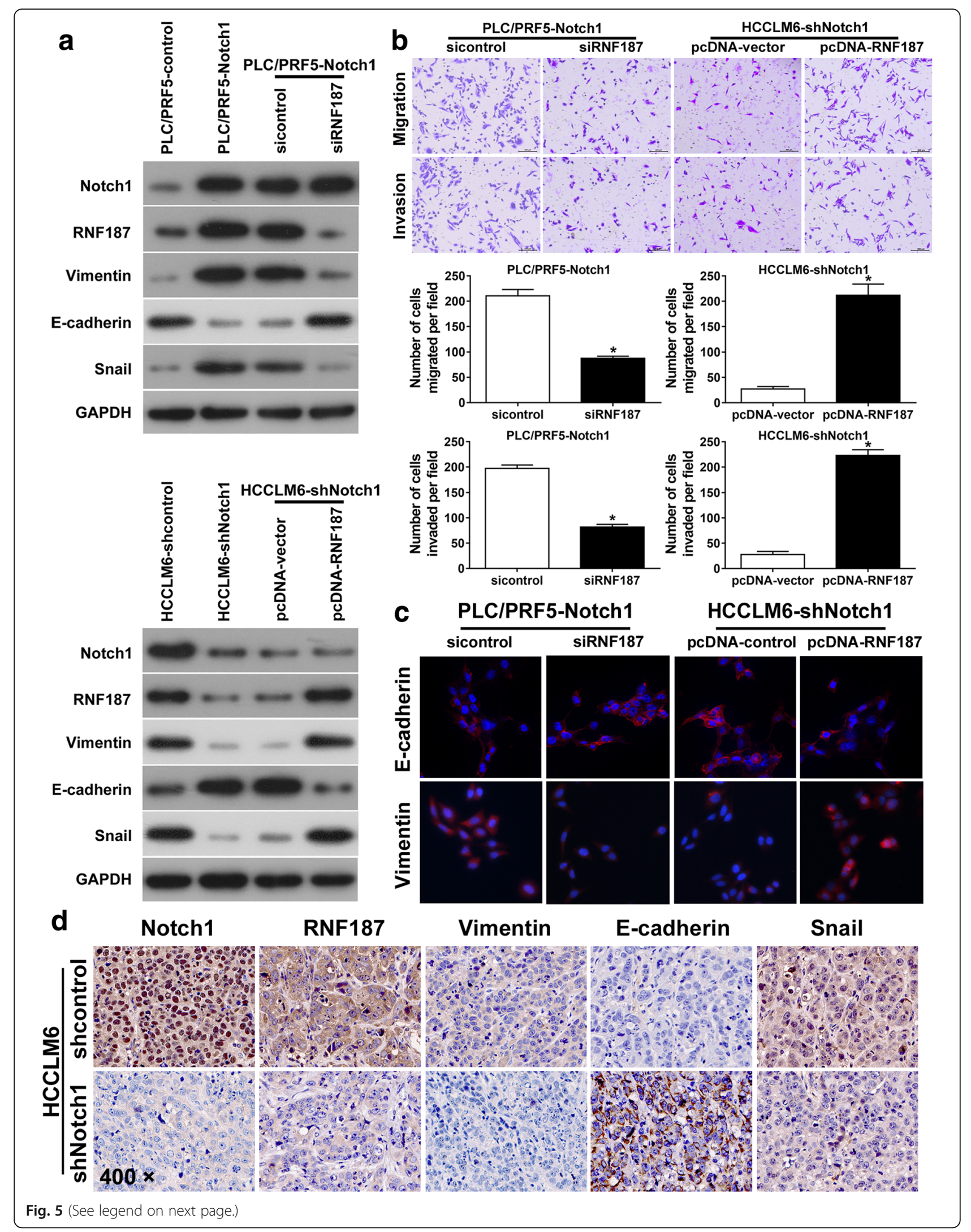


(See figure on previous page.)

Fig. 5 RNF187 is critical for Notch1-mediated HCC migration, invasion and EMT. a Western blotting assays were used to detect the expression of Notch1, RNF187, Vimentin, E-cadherin, and Snai1. b Migration and invasion assays in PLC/PRF5-Notch1 and HCCLM6-shNotch1 cells following RNF187 modulation. c Immunofluorescent staining assay of E-cadherin and Vimentin expressions in PLC/PRF5-Notch1 and HCCLM6-shNotch1 with RNF187 modulation. d Representative IHC images of Notch1, RNF187, Vimentin, E-cadherin, and Snail expression in the xenograft orthotopic liver tumors derived from HCCLM6-shNotch1 and their control cells. *: $P<0.05$. Magnification: $400 \times$

binding sites at promoter regions of RNF187 based on the ChIP-sequencing database (Additional file 6: Figure S4, highlight text, accession no. GSE92701). A luciferase reporter assay showed that Notch1 trans-activated RNF187 promoter activity (Fig. 4g). Sequence analysis revealed 2 putative Notch1 binding sites in the RNF187 promoter. Serial deletion and site-directed mutagenesis showed that the second Notch1 binding sites were critical for Notch1induced RNF187 transactivation (Fig. 4h). A ChIP assay further confirmed that Notch1 binds directly to the RNF187 promoter (Fig. 4i). In our studies, we demonstrated that (1) Notch1 trans-activated RNF187 promoter activity; (2) among the two putative Notch1 binding sites in the RNF187 promoter, the second one was critical for Notch1-induced RNF187 transactivation; and (3) direct binding of Notch1 to the RNF187 promoter was demonstrated by ChIP assay. All these data clearly showed that RNF187 is a direct transcriptional target of Notch1.

\section{RNF187 is critical for Notch1-induced invasion and EMT in $\mathrm{HCC}$}

The overexpression of ubiquitin ligase E3C promoted HCC progression by regulating tumor cell EMT [23]. We assumed that RNF187 plays critical roles in Notch1 pathways effecting HCC cells. To test the assumption, first, we examined the level of RNF187 in Notch1interfered HCC cells. Western blotting assays showed that ectopic expression of Notch1 increased RNF187 expression in PLC/PRF5 cells, whereas Notch1 knockdown inhibited RNF187 expression in HCCLM6 cells (Fig. 5a). To further test whether RNF187 was regulated by Notch1, RNF187-siRNA was transfected into PLC/ PRF5-Notch1 cells, and RNF187 ectopic expression plasmid into HCCLM6-shNotch1 cells. The efficacy was confirmed by Real-time PCR and Western blotting assays (Additional file 7: Figure S5). Western blotting assays indicated that knockdown of RNF187 expression attenuated the loss of E-cadherin induced by Notch1 (Fig. 5a). In contrast, upregulation of RNF187 inhibited the increase in E-cadherin in HCCLM6-shNotch1 cells (Fig. 5a). Then, we measured the migratory and invasive capacity of Notch1-interfered HCC cells with RNF187 regulation. Transwell assays showed RNF187 knockdown decreased the migratory and invasive ability of Notch1 over-expression PLC/PRF5 cells (Fig. 5b). Next, IF assays showed that RNF187 knockdown increased Ecadherin and decreased Vimentin expression in PLC/
PRF5-Notch1 (Fig. 5c). In contrast, upregulation of RNF187 in HCCLM6-shNotch1 cells had the opposite effect (Fig. 5c).

IHC analysis of liver orthotopic xenograft tumor of nude mice revealed that RNF187, Vimentin, and Snail expression levels were higher in tumors derived from HCCLM6-shcontrol cells than in tumors derived from HCCLM6-shNotch1. Whereas E-cadherin expression showed the inverse results (Fig. 5d). Taken the above results together, we concluded that RNF187 is critical for Notch1-mediated migration, invasion, EMT in HCC.

\section{Combination of Notch1 and RNF187 expression predicts HCC prognosis}

We further evaluated the correlation between Notch1 and RNF187 in HCC tissues (Cohort I, Fig. 6a). Both the high expression of Notch1 and RNF187 were associated with aggressive tumor behavior (Tables 1 and 2). Patients were divided into four groups based on Notch1 and RNF187 expression levels. Kaplan-Meier analysis showed that patients with simultaneously high expression of Notch1 and RNF187 had the shortest DFS time (Fig. 6c). Multivariate analysis showed that the combination of Notch1 and RNF187 were risk factors for the DFS time (Table 3). The prognostic value of Notch1 and RNF187 were validated in an independent cohort II of 67 HCC patients by IHC staining (Fig. 6b, Tables 1 and 2). Similarly, the Notch $1^{\text {High }} /$ RNF18 $7^{\text {High }}$ expression patterns had the shortest DFS time (Fig. 6d). Multivariate analysis also confirmed the above findings (Table 3).

\section{Discussion}

Tumor metastasis is a major contributor to HCC patient death. Notch family are critically important in various physiological processes and tumor progression, including liver cancer [11]. In clinical HCC tissues, upregulation of Notch1 has been observed, which is significantly associated with disease progression, such as HCC metastasis $[24,25]$. Our current results provide accumulating evidence that Notch1 played an influential role in HCC metastasis. ChIP-sequencing analysis of Notch1 binding from GEO database indicated 218 genes that may involve in the Notch1 pathway to effect HCC. Among them, OAS2, ATG4C, RNF187, PTMA, and MX2 may potentially be direct transcriptional targets of Notch1. RNF187 is a gene upregulated significantly in response to Notch1 overexpression. Further investigation provided us the 


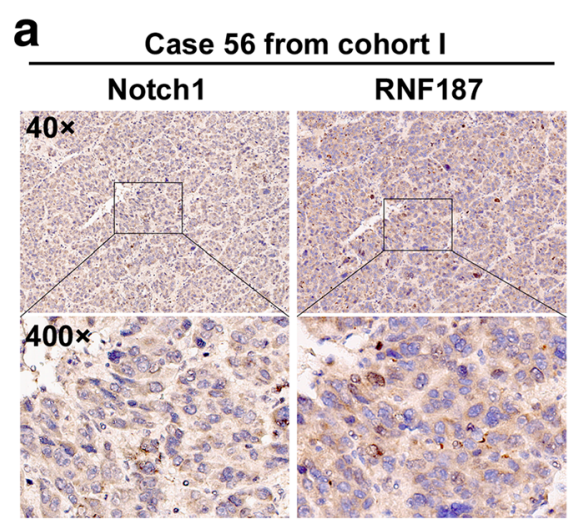

C

\section{Cohort I}

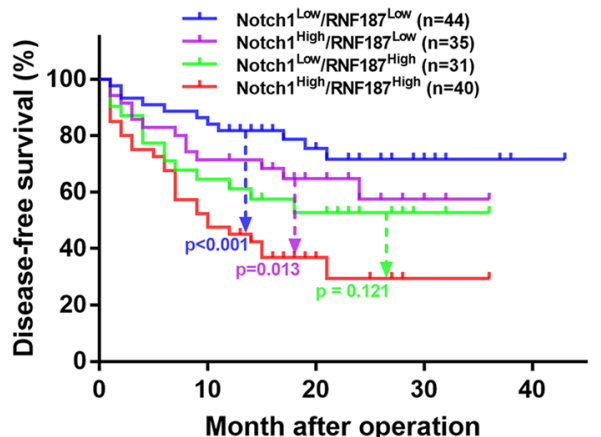

b

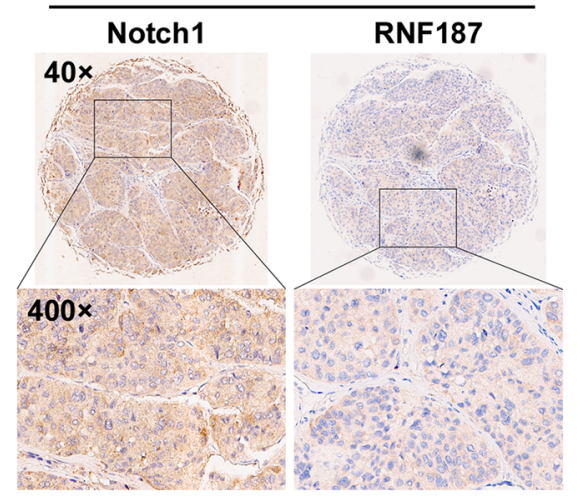

d

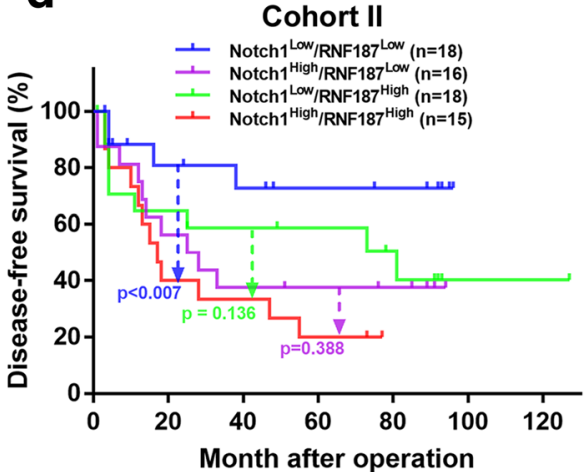

Fig. 6 Patients with high expression of Notch1 and RNF187 had shortest DFS times. a Representative IHC images of Notch1 and RNF187 in consecutive sections from human HCC tissues in the cohort I and cohort II (b). c A Kaplan-Meier analysis of the correlation between the expression level of Notch1 and RNF187, and DFS time in the cohort I and cohort II (d)

Table 2 Association of RNF187 expression with clinicopathological factors of the HCC patients in Cohort I and Cohort II

\begin{tabular}{|c|c|c|c|c|c|c|c|c|c|}
\hline \multicolumn{2}{|c|}{ Clinicopathological variables } & \multicolumn{4}{|c|}{ Cohort I } & \multicolumn{4}{|c|}{ Cohort II } \\
\hline & & \multirow[t]{2}{*}{$n$} & \multicolumn{2}{|c|}{ RNF187 expression } & \multirow[t]{2}{*}{${ }^{a} P$ value } & \multirow[t]{2}{*}{$\mathrm{n}$} & \multicolumn{2}{|c|}{ RNF187 expression } & \multirow[t]{2}{*}{${ }^{\mathrm{a}} P$ value } \\
\hline & & & Low $(n=79)$ & High $(n=71)$ & & & Low $(n=34)$ & High $(n=33)$ & \\
\hline \multirow[t]{2}{*}{ Sex } & Male & 122 & 61 & 61 & 0.172 & 54 & 26 & 28 & 0.386 \\
\hline & Female & 28 & 18 & 10 & & 13 & 8 & 5 & \\
\hline \multirow[t]{2}{*}{ Age, years } & $\leq 50$ & 63 & 34 & 29 & 0.786 & 38 & 22 & 16 & 0.180 \\
\hline & $>50$ & 87 & 45 & 42 & & 29 & 12 & 17 & \\
\hline \multirow[t]{2}{*}{ Serum AFP, ng/mL } & $\leq 20$ & 63 & 35 & 28 & 0.546 & 23 & 6 & 17 & 0.004 \\
\hline & $>20$ & 87 & 44 & 43 & & 44 & 28 & 16 & \\
\hline \multirow[t]{2}{*}{ Tumor nodule number } & Solitary & 123 & 66 & 57 & 0.604 & 52 & 26 & 26 & 0.820 \\
\hline & Multiple & 27 & 13 & 14 & & 15 & 8 & 7 & \\
\hline \multirow[t]{2}{*}{ Maximal tumor size, $\mathrm{cm}$} & $\leq 5$ & 69 & 43 & 26 & 0.029 & 37 & 21 & 16 & 0.274 \\
\hline & $>5$ & 81 & 36 & 45 & & 30 & 13 & 17 & \\
\hline \multirow[t]{2}{*}{ Microvascular invasion } & Absent & 68 & 40 & 28 & 0.169 & 39 & 19 & 20 & 0.695 \\
\hline & Present & 82 & 39 & 43 & & 28 & 15 & 13 & \\
\hline \multirow[t]{2}{*}{ Tumor differentiation } & Well & 19 & 12 & 7 & 0.327 & 22 & 7 & 15 & 0.030 \\
\hline & Moderately or poorly & 131 & 67 & 64 & & 45 & 27 & 18 & \\
\hline \multirow[t]{2}{*}{ TNM stage } & | and || & 97 & 59 & 38 & 0.007 & 47 & 21 & 26 & 0.128 \\
\hline & III and IV & 53 & 20 & 33 & & 20 & 13 & 7 & \\
\hline
\end{tabular}

a Pearson chi-square test was used for comparison between subgroups. Significant results $(P<0.05)$ are given in bold 
Table 3 Univariate and multivariate analyses of risk factors associated DFS of HCC patients in cohort I and cohort II

\begin{tabular}{|c|c|c|c|c|}
\hline \multirow[t]{2}{*}{ Clinicopathological variables } & \multicolumn{2}{|l|}{ Cohort I } & \multicolumn{2}{|l|}{ Cohort II } \\
\hline & $\mathrm{HR}(95 \% \mathrm{Cl})$ & $P$ value & HR $(95 \% \mathrm{Cl})$ & $P$ value \\
\hline \multicolumn{5}{|l|}{ Univariate analysis } \\
\hline Age, years (> 50 vs. $\leq 50$ ) & $0.714(0.437-1.166)$ & 0.178 & $0.939(0.483-1.827)$ & 0.853 \\
\hline Sex (male vs. female) & $1.268(0.701-2.294)$ & 0.433 & $0.696(0.289-1.680)$ & 0.421 \\
\hline Serum AFP, ng/mL (> 20 vs. $\leq 20$ ) & $1.785(1.058-3.011)$ & 0.030 & $0.689(0.352-1.348)$ & 0.277 \\
\hline Tumor nodule number (multiple vs. solitary) & $2.912(1.712-4.953)$ & $<0.001$ & $1.247(0.564-2.759)$ & 0.585 \\
\hline Maximal tumor size, $\mathrm{cm}(>5$ vs. $\leq 5)$ & $5.712(2.978-10.955)$ & $<0.001$ & $1.962(1.006-3.829)$ & 0.048 \\
\hline Microvascular invasion (present vs. absent) & $6.060(3.155-11.643)$ & $<0.001$ & $1.223(0.623-2.399)$ & 0.558 \\
\hline Tumor differentiation (moderately or poorly vs. well) & $12.369(1.714-89.245)$ & 0.013 & $0.879(0.441-1.752)$ & 0.714 \\
\hline TNM stage (III and IV vs. I and II) & $4.739(2.842-7.902)$ & $<0.001$ & $0.865(0.405-1.850)$ & 0.709 \\
\hline \multicolumn{5}{|l|}{ Combination of Notch1 and RNF187 } \\
\hline A vs. D & $0.276(0.136-0.600)$ & $<0.001$ & $0.230(0.074-0.717)$ & 0.011 \\
\hline B vs. D & $0.451(0.231-0.879)$ & 0.019 & $0.680(0.292-1.581)$ & 0.370 \\
\hline C vs. D & $0.599(0.312-1.148)$ & 0.123 & $0.557(0.233-1.332)$ & 0.188 \\
\hline \multicolumn{5}{|l|}{ Multivariate analysis } \\
\hline Serum AFP, ng/mL (> 20 vs. $\leq 20)$ & $1.164(0.664-2.039)$ & 0.596 & NA & NA \\
\hline Tumor nodule number (multiple vs. solitary) & $1.818(1.000-3.303)$ & 0.050 & NA & NA \\
\hline Maximal tumor size, $\mathrm{cm}(>5$ vs. $\leq 5)$ & $2.405(1.085-5.333)$ & 0.031 & $1.914(0.937-3.913)$ & 0.075 \\
\hline Vascular invasion (present vs. absent) & $2.942(1.413-6.125)$ & 0.004 & NA & NA \\
\hline Tumor differentiation (moderately or poorly vs. well) & $3.141(0.395-24.985)$ & 0.279 & NA & NA \\
\hline TNM stage (III and IV vs. I and II) & $1.791(0.928-3.457)$ & 0.082 & NA & NA \\
\hline \multicolumn{5}{|l|}{ Combination of Notch1 and RNF187 } \\
\hline A vs. D & $0.327(0.156-0.685)$ & 0.003 & $0.263(0.084-0.828)$ & 0.022 \\
\hline B vs. D & $0.862(0.425-1.748)$ & 0.681 & $0.884(0.363-2.154)$ & 0.786 \\
\hline C vs. D & $0.769(0.375-1.576)$ & 0.473 & $0.702(0.283-1.738)$ & 0.444 \\
\hline
\end{tabular}

Significant results $(P<0.05)$ are given in bold. Combination of Notch1 and RNF187: A, Notch1 (low) \& RNF187 (low); B, Notch1 (high) \& RNF187 (low); C, Notch1 (low) \& RNF187 (high); D, Notch1 (high) \& RNF187 (high)

evidence that RNF187 was essential for Notch1 to promote HCC metastasis. Notch1-RNF187 association correlated with the prognosis of HCC patients, which might provide a promising strategy for the treatment of Notch1driven HCC metastasis.

Aberrant Notch pathway activation contributes to multisystemic developmental defects and cancer development [8-10]. Notch1 promotes glioma cell migration and invasion through $\square$-catenin and NF- $\kappa B$ pathway [26]. In prostate cancer, Notch1 silence inhibits invasion through matrix metalloproteinase-9 (MMP9) and urokinase plasminogen activator (uPA) [27]. Previous study indicates that Notch1 regulates metastasis of head and neck squamous cell carcinoma by inducing EMT [28]. Herein we reported that Notch1 expression was markedly higher in metastatic HCC tissues than nonmetastasis tissues, and Notch1 expression was correlated with poor DFS, suggesting that Notch1 was a predictive factor for prognosis for HCC. The in vitro and in vivo experiments revealed that Notch1 could promote metastasis of HCC. EMT is a biological process that in which epithelial cells to obtain mesenchymal features, which results in reduced cell-cell contact, leading to increased motility and involving in facilitating metastasis of cancers [29-31]. Though controversial, EMT plays a crucial part in tumor metastasis including HCC [30, 32, 33]. Accumulated evidence implicates that Notch signaling has emerged as a key regulator for EMT [25]. Our study also showed that Notch1 ectopic expression had a significant effect on EMT, as indicated by the decreased epithelial marker expression and increased mesenchymal marker expression. In contrast, Notch1 downregulation showed opposite effects. These findings could be verified by expressions of Notch1, E-cadherin, and Vimentin in xenografted tumors.

Motivated by these results, we presented data regarding a novel Notch1 target gene involved with HCC metastasis: RNF187. In our studies, we demonstrated that Notch1 trans-activated RNF187 promoter activity; and direct binding of Notch1 to the RNF187 promoter was 
demonstrated by ChIP assay. All these data clearly showed that RNF187 is a direct transcriptional target of Notch1. The ubiquitin-proteasome family regulates a series of biological processes including signal transduction and proliferation [34]. The ubiquitination was found to be directly involved in human cancers progression including HCC [23, 35]. RNF187 is a RING domaincontaining ubiquitin E3 ligase. An earlier study reported that in HCC, elevated RNF187 expression was associated with poor clinicopathological features and had shorter DFS, and overexpression of RNF187 resulted in enhanced EMT in HCC [18]. RNF187 is downregulated following nuclear factor kappa $\mathrm{B}(\mathrm{NF}-\mathrm{k} \mathrm{B})$ pathway inhibition in Late Erythroblasts [36]. Overexpression of RNF187 was shown to induce EMT and resistance to apoptosis in non-small-cell lung cancer cells through activation of MAPK and PI3K signaling [19]. These studies indicate that RNF187 is a key player in tumor progression. In this study, we observed that high expression levels of RNF187 was correlated with aggressive clinicopathologic features in HCC patients, including larger tumor size, tumor differentiation and TNM stage. Considering the critical roles of Notch1 and RNF187 expression in HCC tumorigenesis, we aimed to identify the underlying mechanisms responsible for the correlation between Notch1 and RNF187 expression in HCC cells. Luciferase reporter analysis, and ChIP assay confirmed that RNF187 was direct target of Notch1. Notch1-driven HCC progression could be reversed by depletion of RNF187, whereas overexpression of RNF187 counteracted the inhibition of cancer progression mediated by Notch1 knockdown.

In addition, the combination of Notch1 and RNF187 expression predicted prognosis for HCC, further validating the importance of the Notch1/RNF187 axis. The reason why many Notch $1^{\text {High }} /$ RNF187 $7^{\text {Low }}$ patients were seen in our study was due to the statistical method used. In our study, Notch1 and RNF187 were divided into high- and low-expression groups using median cut-off values using the reported statistical methods [37]. As such, half of cases would have low- and the other half would have high expressions of Notch1 and RNF187, resulting in four different combinations in terms of the Notch1 and RNF187 expression levels. Data analysis may be affected by multiple factors, such as sample size, etiologies, ethnic backgrounds, and methods of statistical analysis. Cellular, molecular, and pathological heterogeneity are also important factors contributing to the study discrepancies. In our analysis, discrepancies did exist between the two cohorts in terms of clinicopathological data. However, Kaplan-Meier analysis clearly demonstrated that Notch1 $1^{\text {High }} /$ RNF187 $7^{\text {High }}$ patients had the shortest DFS time (Fig. 6c-d), and this was supported by the multivariate analysis showing that the combination of Notch1 and RNF187 expression was a risk factor for patient survival (Table 3). Thus, both experimental and clinical evidence indicate that RNF187 is essential for Notch1 to promote HCC metastasis.

\section{Conclusions}

In summary, our study clarified a key role of Notch1 and RNF187 in liver cancer metastasis. The results support that RNF187 inhibitors may offer an alternative therapeutic opportunity for HCC patients with high levels of Notch1.

\section{Additional files}

Additional file 1: Supplementary materials and methods. (DOCX $80 \mathrm{~kb}$ )

Additional file 2: Figure S1. The efficacy of Notch1 silence and ectopic expression is determined in HCC cells. (a) Real-time PCR and (b) Western blotting assays confirmation of Notch1 mRNA and protein expression in PLC/PRF5-pcDNA-Notch1 cells, HCCLM6-siNotch1 cells and their control cells. *: $P<0.05$. (TIF $85 \mathrm{~kb}$ )

Additional file 3: Figure S2. The efficacy of Notch1 silence or ectopic expression is determined in stably transfected HCC cells. (a) Real-time PCR and (b) Western blotting assays confirmation of Notch1 mRNA and protein expression in PLC/PRF5- Notch1 cells, HCCLM6-shNotch1 cells and their control cells. *: $P<0.05$. (TIF $98 \mathrm{~kb}$ )

Additional file 4: Figure S3. In vivo lung colonization assays. The indicated stable transfectant cells were injected to nude mice via tail vein. (a) H\&E staining of the lung metastatic tumors. (b) Lung weights, (c) metastatic nodules and (d) metastasis index of nude mice in each group. *: $P<0.05$. (TIF $375 \mathrm{~kb}$ )

Additional file 5: Table S4. DEGs were screened. (XLSX $49 \mathrm{~kb})$

Additional file 6: Figure S4. Prediction of Notch1 binding sites at promoter regions of RNF187. Prediction of transcription factor binding sites at promoter regions of RNF187 based on the Chip-seq database of Notch1 binding from GEO (accession no. GSE92701). Yellow highlight text is binding site 1, and green is binding site 2. (TIF $482 \mathrm{~kb}$ )

Additional file 7: Figure S5. The efficacy of RNF187 silence or ectopic expression is determined in Notch1 mediated HCC cells. (a) Real-time PCR and (b) Western blotting assays confirmation of RNF187 mRNA and protein expression in RNF187 knockdown PLC/PRF5-Notch1 cells, RNF187 ectopic expression HCCLM6-shNotch1 cells and their control cells. *: $P<0.05$. (TIF $97 \mathrm{~kb})$

\section{Abbreviations}

AFP: Alpha-fetoprotein; ANLT: Adjacent non-tumor liver tissue; ChIP: Chromatin immunoprecipitation; DFS: Disease-free survival; EMT: Epithelial-mesenchymal transition; HCC: Hepatocellular carcinoma; IF: Immunofluorescence; IHC: Immunohistochemistry; shRNA: Short hairpin RNA; TNM: Tumor node metastasis

\section{Acknowledgements}

The authors thank Prof. Jian-You Liao and Yu Li, Heng-xing Chen, Zhen Chen, Kai-Shun Hu (Medical Research Center, Sun Yat-sen Memorial Hospital, Sun Yat-sen University) for the help of guidance.

\section{Authors' contributions}

$L X$ and $C L$ conceived and designed the study; $L Z, J C$ and $J Y$ performed the experiments; $L Z$ and $L Q$ analyzed the data and drafted the manuscript; $L Q$, $L X$ and $C L$ revised the manuscript; and all the authors read and approved manuscript.

\section{Funding}

This work was supported by the Natural Science Foundation of China (81672412, 81772597, 81702904); the Guangdong Natural Science Foundation (2017A030311002, 2015A030313033); the Guangdong Science and Technology Foundation (2016A020215199, 2017A020215196); Science and Technology Program of Guangzhou, China (201607010111); Pearl River 
S\&T Nova Program of Guangzhou, China (201610010022); Fundamental Research Funds for the Central Universities of Sun Yat-sen University (17ykpy44); Grant [2013]163 from Key Laboratory of Malignant Tumor Molecular Mechanism and Translational Medicine of Guangzhou Bureau of Science and Information Technology; Grant KLB09001 from the Key Laboratory of Malignant Tumor Gene Regulation and Target Therapy of Guangdong Higher Education Institutes; Grant from Guangdong Science and Technology Department (2015B050501004, 2017B030314026).

\section{Availability of data and materials}

The data and materials of this study are available from the corresponding authors for reasonable requests.

\section{Ethics approval and consent to participate}

Written informed consent was obtained from each patient involved in the study. This study was approved by the ethics committee of Sun Yat-sen Memorial Hospital and Sun Yat-sen University Cancer Center, respectively. All animal experiments were approved by the Institutional Animal Care and Use Committee of Sun Yat-sen University (Guangzhou, China).

\section{Consent for publication}

Written consents for publication were obtained from all the patients involved in our study.

\section{Competing interests}

The authors declare that they have no competing interests.

\section{Author details}

'Guangdong Provincial Key Laboratory of Malignant Tumor Epigenetics and Gene Regulation and Department of Biliary-Pancreatic Surgery, Sun Yat-sen Memorial Hospital, Sun Yat-sen University, 107 Yan Jiang West Rd, Guangzhou 510120, China. ${ }^{2}$ Department of Pathology, Sun Yat-Sen University Cancer Center, State Key Laboratory of Oncology in South China, Collaborative Innovation Center for Cancer Medicine, Guangzhou, China. ${ }^{3}$ Department of Pathology, Sun Yat-sen Memorial Hospital, Sun Yat-sen University, Guangzhou, China. ${ }^{4}$ Storr Liver Centre, Westmead Institute for Medical Research, University of Sydney at Westmead Hospital, Westmead, NSW 2145, Australia.

Received: 7 February 2019 Accepted: 13 August 2019

Published online: 02 September 2019

\section{References}

1. Kulik L, El-Serag HB. Epidemiology and Management of Hepatocellular Carcinoma. Gastroenterology. 2019;156(2):477-91 e1.

2. Yang JD, Roberts LR. Hepatocellular carcinoma: A global view. Nat Rev Gastroenterol Hepatol. 2010:7(8):448-58.

3. El-Serag HB. Hepatocellular carcinoma. N Engl J Med. 2011;365(12):1118-27.

4. Uka K, Aikata H, Takaki S, Shirakawa H, Jeong SC, Yamashina K, et al. Clinical features and prognosis of patients with extrahepatic metastases from hepatocellular carcinoma. World J Gastroenterol. 2007:13(3):414-20.

5. Natsuizaka $\mathrm{M}$, Omura T, Akaike $T$, Kuwata $Y$, Yamazaki $K$, Sato $T$, et al. Clinical features of hepatocellular carcinoma with extrahepatic metastases. J Gastroenterol Hepatol. 2005:20(11):1781-7.

6. Aino H, Sumie S, Niizeki T, Kuromatsu R, Tajiri N, Nakano M, et al. Clinical characteristics and prognostic factors for advanced hepatocellular carcinoma with extrahepatic metastasis. Mol Clin Oncol. 2014;2(3):393-8.

7. Chitnis $A$, Balle-Cuif $L$. The notch meeting: an odyssey from structure to function. Development. 2016;143(4):547-53.

8. Di lanni M, Baldoni S, Del Papa B, Aureli P, Dorillo E, De Falco F, et al. NOTCH1 is aberrantly activated in chronic lymphocytic leukemia hematopoietic stem cells. Front Oncol. 2018;8:105.

9. Stoyanova T, Riedinger M, Lin S, Faltermeier CM, Smith BA, Zhang KX, et al. Activation of Notch1 synergizes with multiple pathways in promoting castration-resistant prostate cancer. Proc Natl Acad Sci U S A. 2016;113(42): E6457-E66.

10. Arcaroli JJ, Tai WM, McWilliams R, Bagby S, Blatchford PJ, Varella-Garcia M, et al. A NOTCH1 gene copy number gain is a prognostic indicator of worse survival and a predictive biomarker to a Notch1 targeting antibody in colorectal cancer. Int J Cancer. 2016;138(1):195-205.
11. Geisler F, Strazzabosco M. Emerging roles of notch signaling in liver disease. Hepatology. 2015;61(1):382-92

12. Morell CM, Strazzabosco M. Notch signaling and new therapeutic options in liver disease. J Hepatol. 2014:60(4):885-90.

13. Chi P, Allis CD, Wang GG. Covalent histone modifications--miswritten, misinterpreted and mis-erased in human cancers. Nat Rev Cancer. 2010; 10(7):457-69.

14. Rape M. Ubiquitylation at the crossroads of development and disease. Nat Rev Mol Cell Biol. 2018;19(1):59-70.

15. Buetow L, Huang DT. Structural insights into the catalysis and regulation of E3 ubiquitin ligases. Nat Rev Mol Cell Biol. 2016;17(10):626-42.

16. Chakraborty A, Diefenbacher ME, Mylona A, Kassel O, Behrens A. The E3 ubiquitin ligase Trim7 mediates c-Jun/AP-1 activation by Ras signalling. Nat Commun. 2015;6:6782

17. Davies CC, Chakraborty A, Cipriani F, Haigh K, Haigh JJ, Behrens A Identification of a co-activator that links growth factor signalling to c-Jun/ AP-1 activation. Nat Cell Biol. 2010;12(10):963-72

18. Yu SL, Wu JC, Liu PF, Liu K, Ye C, Zhou KL, et al. Up-regulation of RNF187 induces hepatocellular carcinoma cell epithelial to mesenchymal transitions. Oncotarget. 2017:8(60):101876-86.

19. Fu Z, Yu W, Wang H, Chen X. Overexpression of RNF187 induces cell EMT and apoptosis resistance in NSCLC. J Cell Physiol. 2019.

20. Meng X, Duan C, Pang H, Chen Q, Han B, Zha C, et al. DNA damage repair alterations modulate $\mathrm{M} 2$ polarization of microglia to remodel the tumor microenvironment via the p53-mediated MDK expression in glioma. EBioMedicine. 2019;41:185-99.

21. Cai J, Chen Q, Cui Y, Dong J, Chen M, Wu P, et al. Immune heterogeneity and clinicopathologic characterization of IGFBP2 in 2447 glioma samples. Oncoimmunology. 2018;7(5):e1426516.

22. Chang RM, Yang H, Fang F, Xu JF, Yang LY. MicroRNA-331-3p promotes proliferation and metastasis of hepatocellular carcinoma by targeting $\mathrm{PH}$ domain and leucine-rich repeat protein phosphatase. Hepatology. 2014; 60(4):1251-63.

23. Jiang JH, Liu YF, Ke AW, Gu FM, Yu Y, Dai Z, et al. Clinical significance of the ubiquitin ligase UBE3C in hepatocellular carcinoma revealed by exome sequencing. Hepatology. 2014;59(6):2216-27.

24. Zhou L, Zhang N, Song W, You N, Li Q, Sun W, et al. The significance of Notch1 compared with Notch3 in high metastasis and poor overall survival in hepatocellular carcinoma. PLoS One. 2013;8(2):e57382.

25. Wang XQ, Zhang W, Lui EL, Zhu Y, Lu P, Yu X, et al. Notch1-Snail1-Ecadherin pathway in metastatic hepatocellular carcinoma. Int J Cancer. 2012;131(3):E163-72.

26. Zhang X, Chen T, Zhang J, Mao Q, Li S, Xiong W, et al. Notch1 promotes glioma cell migration and invasion by stimulating beta-catenin and NFkappaB signaling via AKT activation. Cancer Sci. 2012;103(2):181-90.

27. Bin Hafeez B, Adhami VM, Asim M, Siddiqui IA, Bhat KM, Zhong W, et al. Targeted knockdown of Notch1 inhibits invasion of human prostate cancer cells concomitant with inhibition of matrix metalloproteinase- 9 and urokinase plasminogen activator. Clin Cancer Res. 2009;15(2):452-9.

28. Inamura $N$, Kimura $T$, Wang $L$, Yanagi $H$, Tsuda $M$, Tanino $M$, et al. Notch1 regulates invasion and metastasis of head and neck squamous cell carcinoma by inducing EMT through c-Myc. Auris Nasus Larynx. 2017;44(4): 447-57.

29. Valastyan S, Weinberg RA. Tumor metastasis: molecular insights and evolving paradigms. Cell. 2011;147(2):275-92.

30. Thiery JP, Acloque H, Huang RY, Nieto MA. Epithelial-mesenchymal transitions in development and disease. Cell. 2009;139(5):871-90.

31. Jou J, Diehl AM. Epithelial-mesenchymal transitions and hepatocarcinogenesis. J Clin Invest. 2010;120(4):1031-4.

32. Ding W, You H, Dang H, LeBlanc F, Galicia V, Lu SC, et al. Epithelial-tomesenchymal transition of murine liver tumor cells promotes invasion. Hepatology. 2010;52(3):945-53.

33. Chen L, Chan TH, Yuan YF, Hu L, Huang J, Ma S, et al. CHD1L promotes hepatocellular carcinoma progression and metastasis in mice and is associated with these processes in human patients. J Clin Invest. 2010; 120(4):1178-91.

34. Ravid T, Hochstrasser M. Diversity of degradation signals in the ubiquitinproteasome system. Nat Rev Mol Cell Biol. 2008:9(9):679-90.

35. Bedford L, Lowe J, Dick LR, Mayer RJ, Brownell JE. Ubiquitin-like protein conjugation and the ubiquitin-proteasome system as drug targets. Nat Rev Drug Discov. 2011;10(1):29-46. 
36. Forster L, Finlayson J, Ghassemifar R. RNF187 is downregulated following NFkappaB inhibition in late erythroblasts. Biochem Genet. 2016;54(5):714-21.

37. Han D, Li J, Wang H, Su X, Hou J, Gu Y, et al. Circular RNA circMTO1 acts as the sponge of microRNA-9 to suppress hepatocellular carcinoma progression. Hepatology. 2017;66(4):1151-64.

\section{Publisher's Note}

Springer Nature remains neutral with regard to jurisdictional claims in published maps and institutional affiliations.

Ready to submit your research? Choose BMC and benefit from:

- fast, convenient online submission

- thorough peer review by experienced researchers in your field

- rapid publication on acceptance

- support for research data, including large and complex data types

- gold Open Access which fosters wider collaboration and increased citations

- maximum visibility for your research: over $100 \mathrm{M}$ website views per year

At $\mathrm{BMC}$, research is always in progress.

Learn more biomedcentral.com/submissions 